\title{
Antiviral activity of Cleome rosea extracts from field-grown plants and tissue culture-derived materials against acyclovir-resistant Herpes simplex viruses type 1 (ACVr-HSV-1) and type 2 (ACVr-HSV-2)
}

\author{
Claudia Simões - Tatiana Carvalho de Castro • \\ Lívia da Silva Cordeiro • Norma Albarello • \\ Elisabeth Mansur - Maria Teresa Villela Romanos
}

Published online: 18 September 2009

(C) Springer Science+Business Media B.V. 2009

\section{Erratum to: World J Microbiol Biotechnol}

\section{DOI 10.1007/s11274-009-0147-7}

Unfortunately, in the article "Antiviral activity of Cleome rosea extracts from field-grown plants and tissue culturederived materials against acyclovir-resistant Herpes simplex viruses type 1 (ACVr-HSV-1) and type 2 (ACVr-HSV-2)",
World J Microbiol Biotechnol, Table 3 has been incorrectly published.

The correct Table 3 is presented below:
The online version of the original article can be found under doi: 10.1007/s11274-009-0147-7.

C. Simões · T. C. de Castro - L. da Silva Cordeiro ·

N. Albarello $\cdot$ E. Mansur

Núcleo de Biotecnologia Vegetal, Instituto de Biologia Roberto Alcantara Gomes, Universidade do Estado do Rio de Janeiro (UERJ), Rio de Janeiro, RJ, Brazil

M. T. V. Romanos

Laboratório Experimental de Drogas Antivirais e Citotóxicas

(LEDAC), Departamento de Virologia, Instituto de

Microbiologia Prof. Paulo Góes, Universidade Federal do Rio de

Janeiro (UFRJ), Rio de Janeiro, RJ, Brazil

\section{Simões ( $\square)$}

Departamento de Biologia Vegetal, Laboratório de

Biotecnologia de Plantas (LABPLAN), Instituto de Biologia Roberto Alcantara Gomes, Universidade do Estado do Rio de Janeiro, Centro Biomédico, Rua São Francisco Xavier, 524, PHLC, Sala 509, Maracanã, CEP 20550-013 Rio de Janeiro, RJ, Brazil

e-mail: labplan_uerj@yahoo.com.br 
Table 3 Effective dose to reduce virus titers by $50 \%\left(\mathrm{EC}_{50}\right)$ and selectivity index (SI) of Cleome rosea extracts

\begin{tabular}{|c|c|c|c|c|c|}
\hline \multirow[t]{2}{*}{ Extracts } & \multirow[t]{2}{*}{$\mathrm{CC}_{50}\left(\mu \mathrm{g} \mathrm{ml}^{-1}\right)$} & \multicolumn{2}{|l|}{ ACVr-HSV-1 } & \multicolumn{2}{|l|}{ ACVr-HSV-2 } \\
\hline & & $\mathrm{EC}_{50}\left(\mu \mathrm{g} \mathrm{ml}^{-1}\right)$ & SI & $\mathrm{EC}_{50}\left(\mu \mathrm{g} \mathrm{ml}^{-1}\right)$ & SI \\
\hline In vivo plants (leaves) $(\mathrm{MeOH})$ & 68.7 & 12.9 & 5.3 & 9.3 & 7.4 \\
\hline Pigmented calluses $(\mathrm{MeOH})$ & 4972.2 & 54.2 & 91.7 & 52.4 & 94.8 \\
\hline Pigmented calluses $(\mathrm{MeOH}-\mathrm{HCl})$ & 1535.2 & 43.8 & 35 & 62.6 & 24.5 \\
\hline Acyclovir & $>200$ & $>200$ & 1 & 4.8 & 41.6 \\
\hline
\end{tabular}

$A C V r$ - $H S V$ - 1 acyclovir-resistant herpes simplex virus type $1, A C V r-H S V-2$ acyclovir-resistant herpes simplex virus type $2, C C 5050 \%$ cytotoxic concentration, EC50 effective concentrations to reduce virus titers by $50 \%$, SI selectivity index, Acyclovir standard compound 